\title{
Video Game's design Implications to promote moderate activity in seniors
}

\author{
Raymundo Cornejo, Oscar Ordoñez, Alain Manzo, Adrián Alarcán, Luis \\ Gaxiola, Fernando Martínez, Leonor Duque, Martha Ornelas
}

Published: 30 November 2020

\begin{abstract}
Researchers and different efforts have identified several social, health, financial challenges, among others, with ageing population. Regarding health challenges, the World Health Organization encourages physical activation programs to promote healthy ageing. These programs aim to alleviate imminent musculoskeletal deterioration, or injuries that may occur. Severely increased deteriorations may affect older adults' movement patterns, affecting their autonomy, control, or force. The only way to reduce this deterioration is through physical activation. Therefore, medical gerontology considers physical activity as one of the best tools to improve health and prevent many diseases. Within HCI, research has evaluated exergames as an alternative to engage older adults in physical activation. Exergames have become the technological focus of physical activity, stimulating physical activity through play and competition. However, it is unclear how to maximize these benefits. In this paper we present a set of design implications to improve inter-joint angle for older adults, and maximize activity with its precautions
\end{abstract}

\section{Keywords:}

Exergames; older adults; inter-joint angle; balance; biosignals.

\section{Introduction}

Life expectancy has increased worldwide and by 2050 , expecting we can expect 2 billion of older adults worldwide and from those, 434 million people will be among the oldest old (over 80 years old) [28]. According to the World Health Organization (WHO) [28], 2018 was the first year where people can expect to live 60 or more years. Although Latin American countries are still comparatively young compared with developed countries like Italy, Japan, or Germany [12], ageing will create similar challenges as those in the developed regions of the world.

Oscar Ordoñez, Raymundo Cornejo, Alain Manzo, Adrián Alarcón, Luis Gaxiola, Fernando Martínez, Leonor Duque,

Martha Ornelas

Univeridad Autónoma de Chihuahua

rcornejo@uach.mx a310898@uach.mx amanzo@uach.mx adalarcon@uach.mx lgaxiola@uach.mx fmartine@uach.mx

p325460@uach.mx mornelas@uach.mx
Regardless of the country, governments and social institutions are planning and creating polices to aligning health systems with the needs of older populations, developing systems for providing long-term care, and commitment to healthy ageing [28]. Particularly, active ageing emphasizes the need for action across multiple sectors and enabling older people to remain a resource to their families, communities and economies with the ultimate goal to enhance the quality of life of older adults [26,28]. At the present, the WHO promotes enhancing the quality of life through healthy ageing, which is "the process of developing and maintaining the functional ability that enables well-being in older age" [27]. Functional ability comprises the intrinsic capacity of the person, meaning it entails all the physical and mental capacities of an individual.

Faced with the challenge of accelerated population aging, the European Commission proposed in 2012 the application of a new cooperation framework for innovation in the field of active and healthy aging. The strategic plan adopted then focused its actions on three pillars: 1) prevention, detection and early diagnosis, 2) health care and care and 3) active aging and independent living. For this last pillar, the development of solutions based on information and communication technologies (ICT) that help older people maintain their autonomy, activity and mobility for as long as possible [30] by referring to the European Commission was proposed as a specific action.

In a context common to all developed societies in which the phenomenon of population aging has more and more prominence, it can be understood as an achievement that people reach advanced ages and, at the same time, as a challenge that involves the active approach and satisfactory of this vital stage. The guarantee of aging well will depend on the ability to adapt to the changes that aging brings. Because this adaptation is a protective factor against the loss and decrease of personal resources that every aging process produces in physical, cognitive and emotional functioning [21].

An optimal physical trajectory of an individual across life implies her intrinsic capacity remains high until the end of life. Therefore noncommunicable disease prevention alone lacks of the capacity to promote an optimal physical trajectory in a person, thus compromising her well-being. In their Summative Review [3], Bauman et al. highlight the importance of promoting physical activity among older adults to preserve functional capacity, neuromotor abilities, balance co-ordination, reaction time, and neurological or cognitive health.

Functional capacity and health are an important pillar for the quality of people's social life: the level of functional capacity determines the extent to which they can manage autonomously 
within the community, participate in different events, visit other people, using the services offered by society, and in general, enriching their own lives and that of those closest to them, in addition to older adults is crucial with regard to the way in which they can carry out and support the activities of daily life, which in turn affects your quality of life [7].

Different health organizations recommend physical activity guidelines given the importance of an individual intrinsic capacity in an individual's well-being. For example the U.S. Department of Health and Human Services (DHHS) published a set of physical guidelines for 3 different age groups: children and adolescents, active adults, and active older adults [25]. For older adults, the DHHS recommend:

1. Determine their level of effort for physical activity relative to their level of fitness.

2. Understand whether and how their conditions affect their ability to do regular physical activity safely.

3. At least 150 minutes of moderate-intensity aerobic activity a week or be as physically active as their abilities and conditions allow.

The above requires older adults to possess the knowledge to determine their level of fitness, what are their physical abilities and whether or not they should exercise for more than certain amount of time in one session. Although we acknowledge the above information can be provided to an older adult by a physician, it is important to note how older adults living in less developed countries may not have access to certain health services [20].

Furthermore, activation programs may require certified trainers or older adults may have to go to specific facilities to receive these physical activation classes. Along with these challenges, it is important to consider all different and unique set of abilities for each individual. Therefore, exercises instructions have to be tailored to these abilities to each older adults needs.

HCI and efforts on physical activation have explored exergames for older adults to mitigate the above challenges and promote active ageing. Their results are promising, finding how exergames can be engaging and promote physical activity. Nonetheless, several research effort focused on this but few efforts have explored gaming experienced based on functional capacity and customized movement based on each older adult's physical capabilities, their evolution, and healthcare or biological restrictions.

In this paper, we expand prior research on exergames for older adults by presenting the design and implementation of an exergame aimed to engage older adults in physical activity. Physical activity is one of the main themes given its impact according to Bauman's dimensions and health organizations recommendations. Furthermore, we describe the design ideas learned from an intervention with specialists and older adult. The design ideas lead the design of such exergame which integrates physical and biological analysis to customize the gaming experiences according to the older adults' physical capabilities. Furthermore, this customization is aimed to continue the older adults' progress with their inter-joint angle and balance by raising the level of each minigame. Finally we present the conclusions and future work towards the broader evaluation with older adults to promote their physical activation.

\section{Related Work}

Over the years, several research has been conducted to support therapies for the physical rehabilitation of an older adult. Medical research have studied which physical aspects correlate to a better state of well-being through the analysis of therapy information. HCI research have also explored several technologies to support therapy sessions, the use of exergames is one of such technologies which has proved to be engaging for older adults despite if they are tech-savvy or not. Therefore to situate our work, we consider previous research conducted in the areas of Physical activation, and exergames, detailing inter-joint angle and balance as two key aspects on physical activation and the acceptance of exergames by older adults.

\subsection{Inter-Joint Angle and Balance}

Daily exercise contributes to maintaining older adults' physical capability, which manages to maintain their functional abilities in terms of motor skills and activity level, also helping to prevent falls and accidents. Regardless whether the older adult is involved in a rehabilitation context, or not. [37].

Researchers [17,34] have studied specific inter-joint angle, such as movements of the upper extremities. The latter is very important to develop the activities of our day to day, p.e. lifting objects, participating in some physical activity, and even performing tasks for proper self-care (eating, grooming) [17].

The use and application of the virtual model for the rehabilitation of upper limbs in older adults will help in physical activity since it allows measuring the angles of joint mobility with respect to the optimal angle, the data generated is more valuable for the physical educator and at the same Easy time to visualize because the patient will only need to stand in front of the sensor. In addition to being able to analyze patients with trauma disorders, it is possible to use this system in patients with joint deterioration and other degenerative osteomuscular pathologies. With the use of new technologies represented in various formats it is possible to detect the orientation of the arm, so that the pronation and supination movement could be implemented giving the opportunity to include more movements to study [40].

Additionally, throughout our lives, it is common for our physical abilities to decay. Events such as falls are responsible for increasing mortality, and exposing older adults to fractures, damaging their quality life. The most efficient way to improve balance and reduce the risk of falls in older adults is by physical activity [13].

Approximately $30 \%$ of adults over 60 could experience a fall during their lifetime. Falls are considered the second cause of death due to accidental injuries in the elderly population and the decrease in physical condition at this age is usually one of its triggers [31].

\subsection{Exergames for Older Adults}

Through the history of the video game industry, a variety of input devices had been developed to involve the player into a new and more active role in the game. The release of new consoles have contributed to the growth of video game players' community. This growth includes people in their forties or fifties [1, 8, 16, 43]. In addition, studies suggest the Wii console had a very positive reception on an audience composed of independent older adults and older adults living in retirement communities [16,18,24].

Sato et al. [37] presented a video game with a full body interaction using a Microsoft Kinect sensor, immersing players in $3 \mathrm{D}$ environment with the Unity framework. Authors conducted a randomized controlled trial with 57 healthy elderly individuals and their results indicate a positive impact on walking, muscle strength, and motor function with participants in the intervention group (see Figure 1). Similar studies have explored the possibilities of improving the balance through the use of the Kinect sensor. In [14], 
researchers developed a game based on stepping exercises to provide older adults with adaptive training to aim at reducing the risk of falling. The video game was designed to be used in a clinical environment and their results were favorable but further assessment is needed to observe clear positive outcomes.

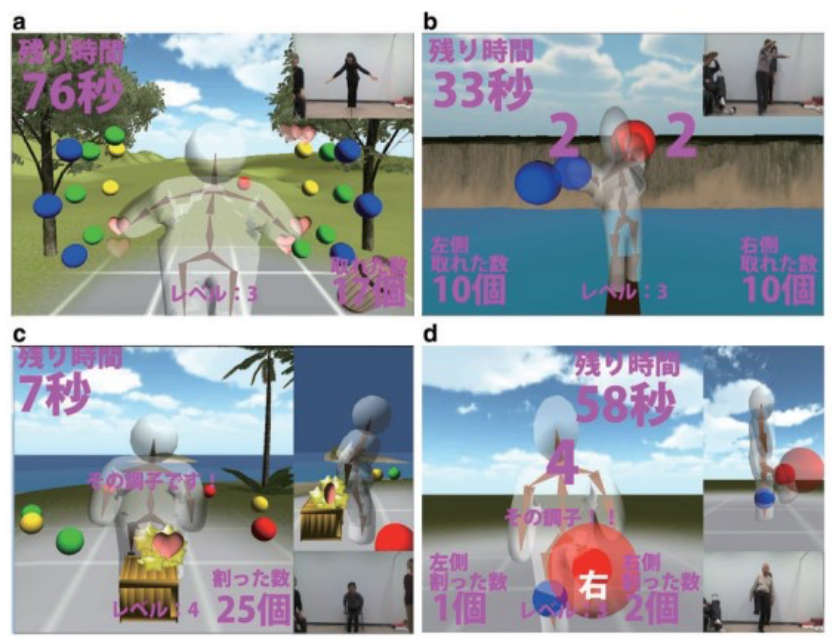

Figure 1. Exergames' screenshots based on full body sensing to improve walking, muscle strength, motor function, from [37].

With the addition of video game accessories, such as the Wii balance, researchers have also design, developed, and evaluated video games with an exertion approach that is inclusive for players, such as older adults. This inclusion has led to motivate older adults to perform physical activities to improve their balance and reduce risks of falls $[1,16,43]$.

SilverBalance [16] is a minimalist and adaptive prototype using Wii balance. Game difficulty adaptation is set by each user, and the main objective of this video game is to promote balance activities. In their results, researchers strongly suggest the benefits of using the above technologies to offer new forms of physical activation (see Figure 2). In a similar manner, Bateni et al. [2] found empirical evidence on how video games may improve the balance of older adults. However, using the physical Wii board implies older adults have to be exposed to a potential fall since these technologies lack of physical aids to prevent falling.

Gerling et al. [15] proposed a set of guidelines to design exergames for older adults which employ full body sensing capabilities. Among these guidelines, Gerling et al. propose to a dynamic gaming difficulty to adapt to the older adult's range of motion. The proposed adaptation is based on a calibration sequence that determines the level of gesture precision needed to play. In a similar manner, Velázquez et al. [41] propose a set of four recommendations for adapting the exertion experience in exergames. These recommendations focus on the older adults' motion capacity and perceive exertion.

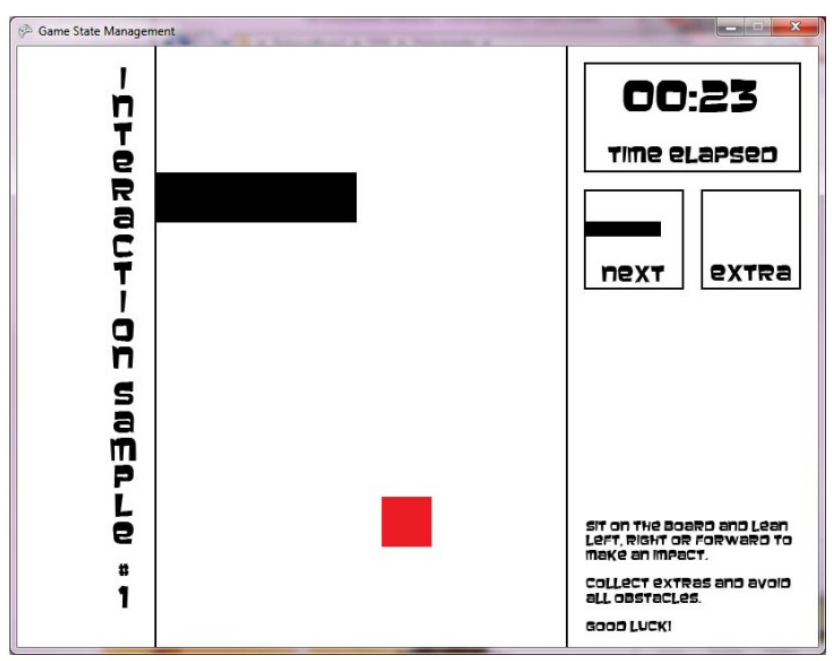

Figure 2. SilverBalance, an exergame base on "different balance tasks for elderly players featuring the Nintendo Wii Balance Board", from [16].

In addition to promoting physical activity in the elderly, serious video games have been developed with other objectives. One of these is Guess My Caption [10], which in addition to providing physical rehabilitation through body movements as a way of interacting with the game, seeks to strengthen the bond of the elderly with their family members (see Figure 3 ). Although the results did not show significant changes in the increase of arm strength after using the serious video game, the older adult stated in the interviews that he felt improvement in his physical condition, suggesting that serious video games can change the perception of the elderly in relation to their health. In this serious video game, no real-time adaptability was implemented according to the physical characteristics of the elderly.
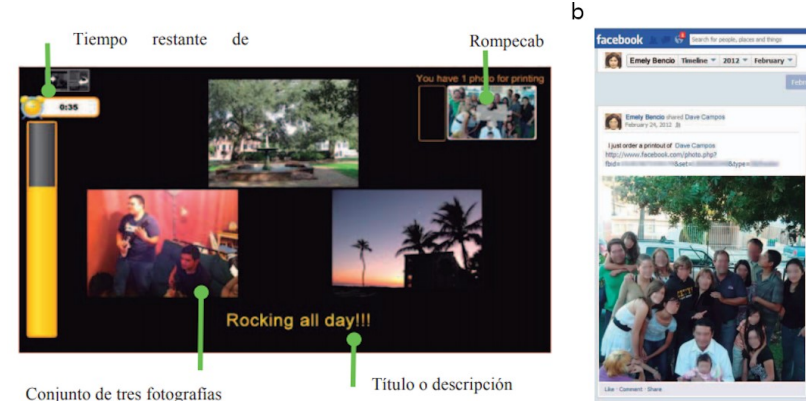

Figure 3. Guess My Caption, a movement-based exergame considering socialization as a design factor to have a positive impact on elderlies' well-being perception, from [10]. a) Exergame's screenshot, b) Automatic social media post as a player reward.

As stated above, there has been evidence on the benefits of using exergames as an intervention to promote physical activity with older adults. We argue similar design recommendations as well but build on top of these and present new design recommendations to not only engage older adults but ensure a moderate physical activity to contribute to their health and wellbeing. 


\section{Design Methods}

Our methods followed a user-center approach and were conducted with a multi-disciplinary research team and older adults Northern Mexico. Over a period of four months we conducted design meetings and a focus group. The aim of our qualitatively inquire was aimed to understand further the requirements and challenges to support physical activation and older adults' perspective towards video games.

\subsection{Design Meetings}

The aim of these meetings were to have a better understanding of the inter-joint angle and balance of an older adult. Including traditional techniques to obtain this information. As well, our inquiry focus on determining the expected intensity to carry out the physical activities and have an impact in health and well-being.

Over four months, eight meetings were conducted with experts from different areas. Each meeting had a duration between 60 to 80 minutes. At each meeting, notes were taken to organize the topics covered in the current and future sessions. Also, audios of 3 sessions were recorded for further analysis and consultation. The meetings were attended by a multi-disciplinary research team divided in two main areas: computer science and physical health science. The research team consisted in a signal processing expert, an evolutionary algorithm expert, a software architect with 14 years of experience, an expert in human-computer interaction, a motor development researcher, a Physical and functional Physician, a Physical and functional Physician for older adults with disabilities, and a Physical and functional Physician for independent older adults.

An additional session of physical exercises was held with the members of the research team. This session was conducted to complement our understanding from the meetings and have a firsthand impression on which type of exercises should be done by older adults. This session was also audio and video recorded.

All the data from above was obtained with the prior consent of all the participants.

\subsection{Focus Group}

We conducted a focus group meeting to investigate the perspective of older adults towards video games. In this focus group, three female older adults, whose ages are between 70 and 78 years old, shared their opinions and thought about video games. The session was conducted at the Faculty of Physical Culture Sciences buildings located in Chihuahua, Chihuahua, Mexico. For further analysis and consultation, the focus group participants agreed the session could be audio recorded. The session consisted in two phases: exploration of themes, and demonstration of lowfidelity prototype.

During the exploration of themes, two members of the research team posed questions to offer an opportunity for dialogue. The questions were designed to explore the relationship of older adults with video games. For example, we inquire if the older adult is or was an active video game player. In addition, we also investigate the direct or indirect interaction older adults may have with video games through relatives or friends.

Based on this general theme, we also explore themes such main impediments older adults face to participate actively in video games, or understand older adults' main motivation they do play video games.

On the second phase, the two research members showcased a low-fidelity prototype of a video game. The prototype design was based on the literature review and findings from the design meetings. One research member presented all the interaction with the video game through mimics and simple representation of the game scenes. This was conducted in this way to receive feedback from participants such as design improvements. Furthermore, this sessions allowed us to adjust design metrics and make future decisions, such as finding the right way to give instructions on how to carry out an activity. Finally, we conducted a brainstorming session to motivate and engage the older adult.

We analyzed the data collected by following a constructionist grounded theory approach [9].

Therefore, our findings emerged through interactions with our data, and between members of our research team. Research members analyzed and compared the data collected, and discussed the emerging themes to seek further refine and explicate our emerging understanding of physical therapy, older adults, and video games. Through this iterative process, we began to view the dimensions and design ideas to support video games for older adults and physical activation.

\subsection{Findings}

From our analysis, we established four implications for the design and development of an exergame focused on physical activation targeted to older adults: 1) exergames must consider players who may suffer from physical or cognitive disabilities, or even both. Video games must be able to adapt to the player physical abilities and capabilities. One way to do this is by offering a dynamic difficulty that changes based on the player performance throughout the game. 2) Include activities according to the range of inter-joint angle of upper and lower limbs. In older adults, the range of motion gets reduced, i.e. the full-body interaction is limited compared to their younger peers. An adjustment of the game activities to the older adult capabilities can prevent injuries while playing, that is, a previous and ongoing calibration may be necessary to know the player range of mobility. 3) Determine older adult's max level of intensity which the player can perform the activity and reduce the level to moderate. 40 - 60\%. Moderate intensity is considered the indicated level to benefit the health of physically active individuals. Use biological signals to determine the moderate level, for example, heart rate (HR), heart rate variability (HRV), and stiffness index (SI) in addition to the galvanic skin response can be calculated to determine if the player performance in an exercise is with a moderate and constant physical intensity. 4) Clear instructions and continuous tutorials. In order to avoid injuries and other accidents, is very important to settle down the right way to present instructions. Thanks to the focus group, it was determined that the best way for older adults to receive indications is the combination of a voiced instruction with a quick demonstration. The last one can be achieved through mimics.

Conceptualization of motor stories were set through the analysis of the information collected during the second phase of our focus group. The stories reflect everyday life activities in which the player knows how to perform them, i.e., painting a fence, hitting a pinata, or placing Christmas arrangements. Incorporating everyday activities into ludic interactions creates low cognitive demand tasks since they are activities a player may be familiarized with. Our participants express interest in motor stories' potential to provide extraordinary stories, e.g., exploring pyramids, picking apples in a vegetable garden, or diving. Maintaining the interest of the player in the physical activities.

\section{Design and Implementation}

The functional prototype was developed, considering our four design implications gained in the findings of the above section. 
The following sections describe the game's narrative and how its design and interaction take into account our design implications. Next, we describe how the video game calculates a set of biological signals to adjust the video game's difficulty.

\subsection{Exergame Design}

The video game's difficulty on the dynamics and form of interaction could create a steep learning curve for the conventional older adult. This leads to a low adoption and engagement of older adults with video games. Therefore, we followed our first design implication to be incorporated, as a high priority, a natural interface and an adaptive difficult mechanism. As a natural interface we chose to implement a Full-Body Motion-Based Interaction using the Kinect v2 to avoid the use of traditional controllers, i.e. joysticks, that require a finer hand-eye coordination. On the other hand, the main goal of the adaptive difficult mechanism is to reduce the learning curve by offering the older adult physical challenges according to their physical capabilities. Therefore, the video game adjust the difficult considering the inter-joint angle between the upper limbs and the torso, and the inter-joint angle between thigh and pelvis. As one of the first activities is a calibration process to recognize the older adults physical constraints (see Design implication 2). Knowing the physical limits of the player can provide a personalized experience. A series of short poses will achieve the calibration process and store the motor skills of the player.

Current clinical inter-joint measurements techniques are performed by a physician with a goniometer. The nature of this tool implies an invasive procedure where the patient has to lie down or stand-up in order the physician takes the measurements. Therefore, the FullBody Motion-Based Interaction of the video game allows to avoid this procedure and take reliable measurements [35], obtaining older adult's mobility range and follow our second design implication. Furthermore, the adaptive difficult mechanism can use the Kinect measurements in real time, without the need to invade the patient, adjusting the video game activities to the older adult's capabilities and record any progress in their mobility range without the need of a physician or goniometer.

It is well documented users tend to lose interest in conventional physical activities because they considered them boring and demotivating [13]. This is no exception for older adults. Therefore, it is important to provide them ways to get engaged in participating in the exergame.

Our video game incorporate a set of motor stories to sustain the interest of the older adult in the physical activities. The stories reflect everyday life activities in which the player know how to perform them, e.g., painting a fence, hitting a piñata, or placing Christmas arrangements. Incorporating everyday activities into the ludic interaction create a low cognitive demand tasks since they are activities a player may be familiarized with.

In addition, motor stories have the potential to provide extraordinary stories, e.g., exploring pyramids, picking apples in a vegetable garden, or diving. Thus, maintaining the interest of the player in the physical activities.

Figures 4, 5, 6, 7, 8 are screenshots of activities from a motor story from the designed video game. For each one, the player is given instructions through mimics (according to our fourth design implication).

The motor story represents a birthday party where the older adult has to complete five activities related to the preparation and participation of the party. This motor story exemplifies the design of the video game. Our focus group widely accepted this scenario, commenting that the activities are familiar and entertaining. As the
Figure 4 shows, the player is placed in front of a wall, where s/he must reach the blue spheres and replaced them by red cubes. These cubes represent part of the party decorations. This motor story aims to work the upper body inter-joints angles.

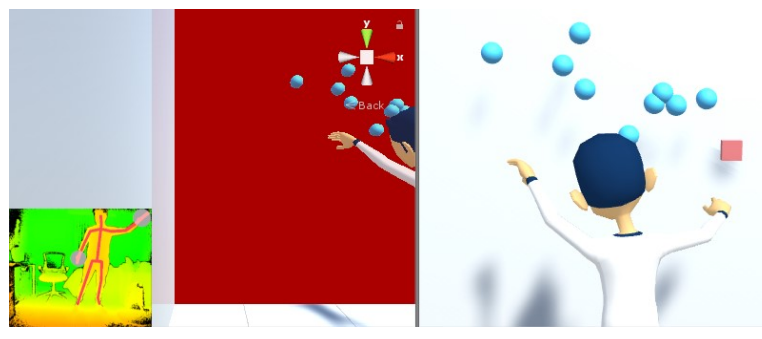

Figure 4 Screenshot of decorations placement motor story, by reaching blue spheres with upper body inter-joints activity.

For the next activity, the player is placed into a room representing a kitchen where a cake has to be baked, see Figure 5. The player must catch different size and color discs that will fall from the ceiling. The player movement is achieved through lateral displacements. These discs represent the floors of a birthday cake. The speed, frequency and opening angle with which these discs are released are adjusted according to the player's performance during the game (see First design implication).

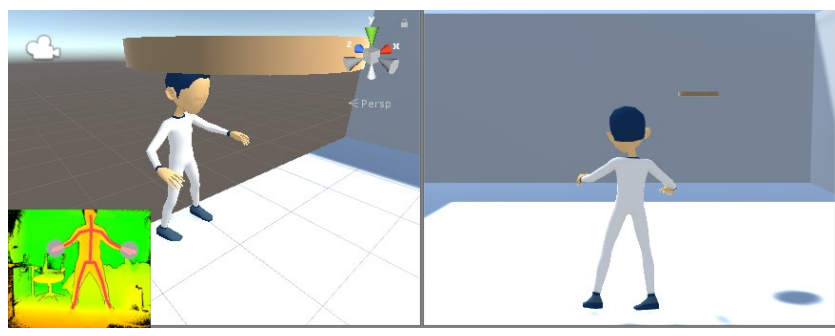

Figure 5 Screenshot of motor story with the aim of catch discs (cakes) through lateral displacement.

A gift scene comes next. The player must raise their arms pretending to carry a gift (see Figure 6). The avatar representing the player will move in a straight line when the player lifts each knee. The knee movement is performed by alternating each leg and trying to achieve a 90-degree inter-joint angle. This is done repeatedly until the avatar reaches the end of the room, where the box is automatically placed and the avatar returns to the initial position. Following our first three design implications, the video game adjusts a valid angle between the $40-60 \%$ capacity of the player.

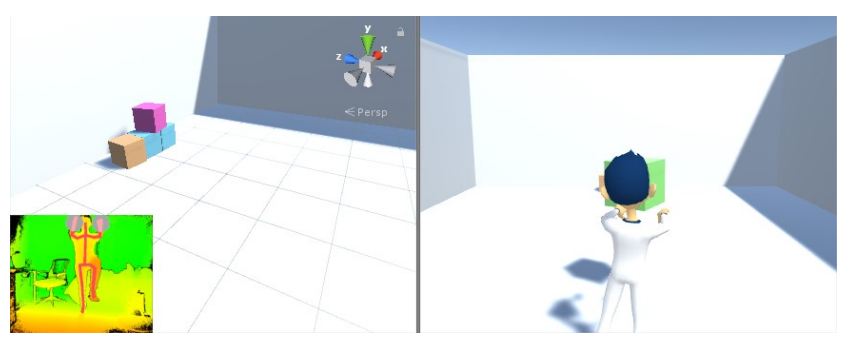

Figure 6 Screenshot of gift arrangement motor story, with both upper and bottom body inter-joints activity. 
In accordance to our users traditions, we introduced an activity where the player has to break a piñata (see Figure 7). Within this activity the avatar has a stick with which can hit the piñata. The piñata will break a certain number of hits, customized to the strength of a player (i.e. the video game detects the older adult starts to low her arms due to fatigue). The piñata drops candies, represented by spheres, and the player has to pick up as many as $\mathrm{s} /$ he can. This dynamic is similar to the first activity presented in Figure 4 where the player stands up and uses the upper limbs to collect the candy. Both activities consider our design implications to provide a custom experience.

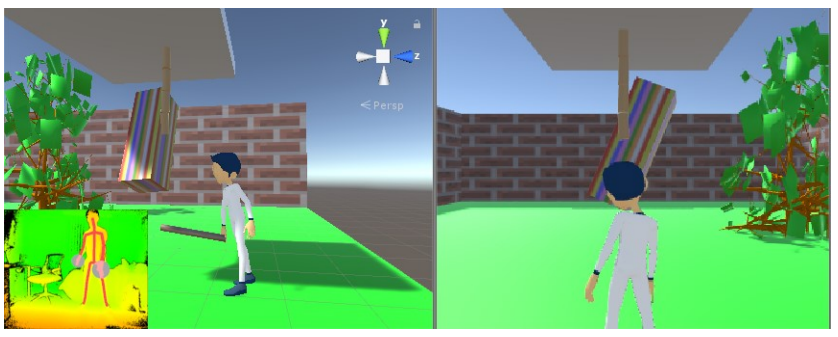

Figure 7 Screenshot of the piñata motor story.

As part of the motor stories and engagement, it was important to understand the type of video game rewards of interest to an older adult. Our analysis indicates virtual rewards are viable for older adults to continue participating. These rewards differ by the individual interest of each participant but converge into virtual rewards for the avatar or a private virtual room, For example, a player can obtain accessories to customize the avatar of each player. These accessories can be obtained from accomplishing the motor stories. Furthermore, virtual rooms can represent virtual gardens in which you can customize or a room of virtual trophies in which you can place photographs of the player while playing. In addition to rewards, we designed new motor stories based on the themes explored with the focus group and their suggestions, ideas, concepts, and opinions about video games.

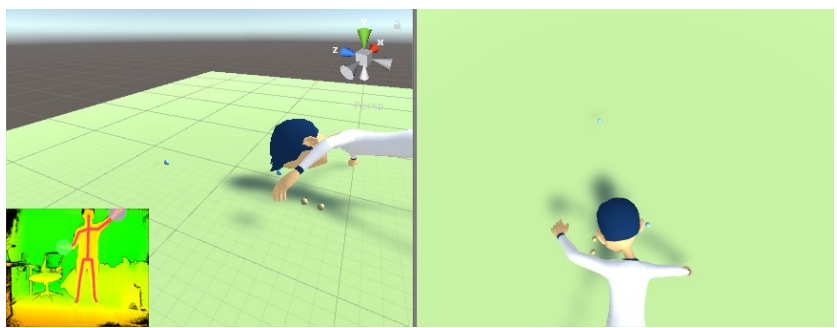

Figure 8 Screenshot of motor story with the objective of reaching colorful spheres with upper body inter-joints activity.

The player's performance will be measured during the duration of all the motor stories. From our design meetings, the experts in human mobility area recommended the older adults should perform activities with a moderate intensity of $40-60 \%$. This intensity will have to be as constant as possible for a period of 1520 minutes (see design implication 3). Biosignals such as heart rate (HR), heart rate variability (HRV) and stiffness index (SI) can be measure to determine the intensity carried out in an activity. This information can be shared in real time with the therapist and even the player and adjust the activity difficulty. Therefore, our design can ensure the effectiveness of the exercise by having reliable information of the moderate activity.

The video game implementation incorporates a group of technologies to support the real time experience between all hardware and software components used in this project. The architecture is composed by 5 main subsystems: NodeJs, Kinect v2, Unity, OpenBCI in two layers, and local and cloud Infrastructure (see Figure 9). First author designed the video game over Unity $3 \mathrm{D}$, all-in-one platform for game development which provide tools and services and rendering $2 \mathrm{D}$ and $3 \mathrm{D}$ images.

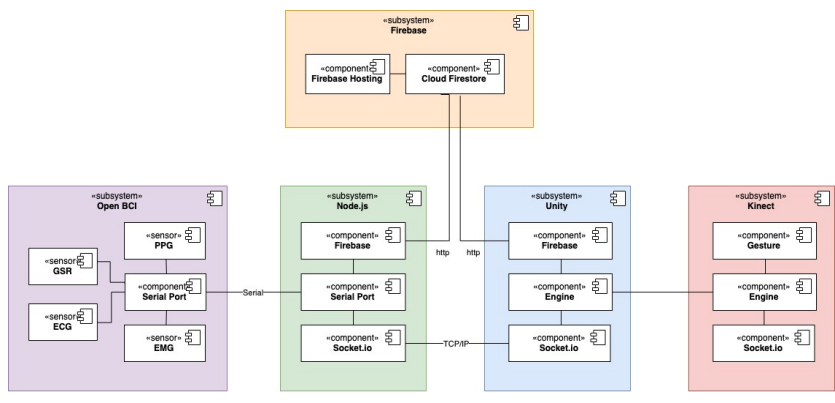

Figure 9 Architecture component diagram.

We used a heart rate, and pulse sensors in a OpenBCI Cyton board to get, store, and process the physical activity data in real time. The data parser and manager were implemented in a NodeJs backend which processes all the information we are gathering from the sensors. All the information we are collecting from the video game is also sent to the backend over TCP/IP protocol using Sockets as a real time communication protocol.

The information is stored in a real time NoSQL dataset synced in real time. Furthermore, we developed a single web page application to monitor the activity with a 3-way data binding via Angularfire with a minimal setup and easy access to data, files, auth, and highly secure. The implementation to monitor vital signs is described in the following section.

\subsection{Bio Sensing}

We analyzed vital signs along with some biosignals as indicators to assess the cardiovascular status of the players. This monitoring prevents an older adult from over-exerting since the exergame can adjust the speed or difficulty of the game. The video game can even stop if the state of health is threatened or if it goes beyond the levels recommended by the specialists. Common vital signs are heart rate, breaths per minute, and blood pressure.

In this work, we are focused on monitoring both, electrocardiogram (ECG) and photoplethysmographic signal (PPG) for analyzing the heart rate (HR), heart rate variability (HRV) and stiffness index (SI). The hardware used for the task described above is based on the OpenBCI Cyton Board, which it has an 8-channel neural interface with a 32-bit processor, communication via Bluetooth, 3-axis accelerometer, Micro SD card slot and 5 GPIO pins, 3 of which can be Analog (see Figure 10). We use three passive gold electrodes to register the ECG activity and a standard electrode paste to adhere the electrodes to the body. Basically two electrodes have to be connected to any of the eight channels of the OpenBCI Cyton board, while the third electrode should have a connection to the BIAS terminal. In addition, we used a pulse sensor to register the PPG signal, where the signal wire of this 
sensor is connected to any of the three analog pines (pin D11 in our case) on the board.

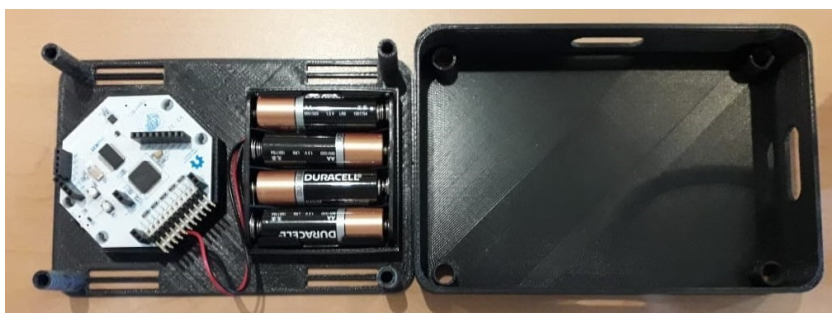

Figure 10 OpenBCI Cyton board and accessories for monitoring biosignals.

We use OpenBCI software to have a first test on the register of the biosignals. The connection of the electrodes to the body have a configuration leftwrist, rightwrist and rightankle. In the case of the pulse sensor, it is set on the leftearlobe. The design of the video game involves different types of analysis about the cardiovascular condition. The video game contains a layer (algorithms) for carrying on these analyses in real time. This layer captures the biosignals from the OpenBCI Cyton board using serial AT commands. Next section describes the methods for compute the different types of analyses.

\subsubsection{Analysis of the cardiovascular status}

Analysis of Heart Rate helps in diagnosis and detection of coronary diseases and it is considered a non-invasive indicator of illness. An elevated resting HR has been associated with cardiovascular and all-cause mortality, independent of major cardiovascular risk factors, and with cancer mortality [36]. Generally, ECG signal is used to compute HR, however, in this work we used PPG signal instead. PPG represents the variations in blood volume in the body and it is synchronous to the heart beat, so it can be used to detect heart rate. We compute HR using [36], where $\mathrm{T}_{\mathrm{bb}}$ is the time that elapses between each maximum of PPG signal.

$$
H R=\frac{1}{T_{b b}} * 60
$$

The variability of the heart rate represents how much the period between heart beats varies [39]. The rhythm of the heart is controlled by the sinus node, which is modulated by nerve endings of the parasympathetic and sympathetic system. Sympathetic activity tends to increase the heart rate and its response is slow (few seconds). On the other hand, parasympathetic activity tends to decrease the heart rate $(0.2$ to 0.6 seconds) $[32,38]$. HRV can be measured by different techniques, we use the one that is based on the spectral methods. These methods start from the information thrown by the periodogram (see Figure 11). The periodogram is the graphic representation of the heart beats over time, and the information for this representation is obtained from the electrocardiogram. In spectral methods the most outstanding component of the HRV is the arrhythmia of the respiratory sinus, which is considered in the range of 0.15 to $0.4 \mathrm{~Hz}$. In addition to the physiological influence of respiration on $\mathrm{HRV}$, this high frequency component (HF) is usually associated with an influence of the parasympathetic system [32]. Another studied component of $\mathrm{HRV}$ is the low frequency component (LF), usually in the range of 0.04 to $0.15 \mathrm{~Hz}$. The spectrum within the LF band is attributed to sympathetic activity as parasympathetic, however, some researchers have suggested that the influence is mainly of sympathetic origin [38].

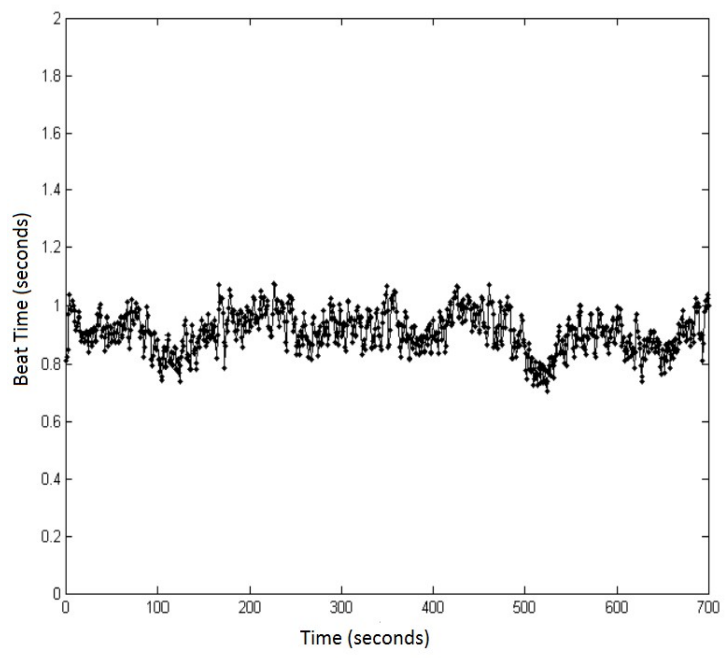

Figure 11 Periodogram of a healthy volunteer in a resting state (sitting).

HRV has been studied extensively during the last decades, its use has served to predict risks after a myocardial infarction, as a preventive diagnosis of diabetic neuropathies and of suffering cardiac attack. In addition, it has been found that HRV can give important information about age, physical and mental stress $[32,38,39]$.

Stiffness arterial is recognized as a cardiovascular risk factor and represents a mayor index of tissue damage [11,19,22], where aging produces alterations in the composition and distribution of elastin and arterial collagen, which explain the stiffness or less distensibility of the arterial system and the increase in peripheral vascular resistance $[4,33]$. As a consequence, a lower elasticity of the blood vessels, the systolic blood pressure increases and the diastolic blood pressure decreases, in this way, the arterial stiffness can increase passively each time the blood pressure rises, in addition of structural changes induced by age. The stiffness index (SI) in $m / s$, is defined as the ratio of the patient's height (h) divided by the transit time $\Delta \mathrm{T}$ according to [23]. The transit time is defined as the time between peak and peak (maximums) of the PPG signal (see Figure 12). When the second peak (known as a dicrotic node) of the PPG signal does not exist, the inflection point is usually used.

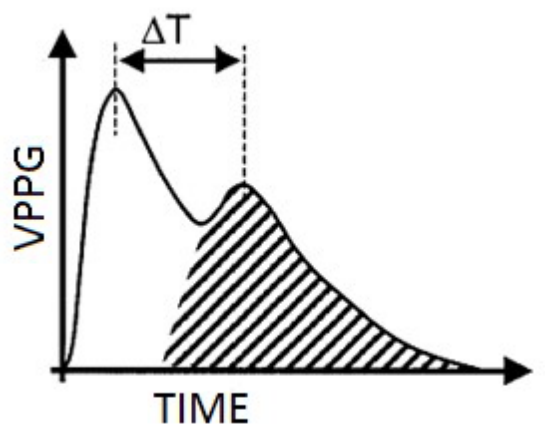

Figure 12 Characteristic morphology of the PPG signal. 


\section{Discussion}

In this paper, we present a set of implications for design and the implementation of an exergame for older adults. We acknowledge prior work on this regard; however, our contribution can be seen in two main aspects: an extension on prior design implications where moderate physical activation is achieved through the analysis of vital signs, and the design and implementation of one exergame that follows this set of implications. This exergame incorporates motor stories to represent familiar activities to older adults and encourage them to perform physical activities with the aim of improving their motor capacity. In addition, our design incorporates a bio-sensing system to monitor and capture vital signs of older adults while playing. This system monitors electrocardiogram (ECG) and photoplethysmographic (PPG) signals to analyze the heart rate (HR), heart rate variability (HRV) and stiffness index (SI). Monitoring these vital signs can ensure a safe environment but also promote a moderate activity. This is particular important since health physicians recommend older adults to do exercises with moderate activity in order to have a positive impact on their well-being. We expect these implications could evolve into guidelines that promote the future design of exergames that could improve the mobility of older adults and their health with a ludic and entertaining experience.

The advantage of using this type of visual and bio sensors, unlike other video gaming consoles, is the ability to process video footage in real time and capture movements of different parts of the user's body. For example, Wii consoles could only monitor the movement the user's hands and with our system we could infer the full range of articular movement and their physical health. In addition, system reacts optimally to user movements without the need for set special lighting or privacy conditions since the depth camera can be programmed to follow and recognize only people of interest in different scenes, regardless of the rest of the objects present in the environment [6].

The use of serious video games as a tool for physical activity is part of a worldwide trend marked by the use of new technologies in increasingly diverse environments. The increase in the so-called technophilia can become an especially attractive alternative in vulnerable populations such as the elderly. For a personalized health perspective, the games provide a new source of information about the user, their preferences, skills, abilities and their style of play. Technological sensors such as Kinect combine with biosignals monitoring allows the collection of data related to the kinematics of each player, heart rate, and other gaming themes such as game scores, time spent for each activity or even energy expenditure [29].

Older adults who do regular physical activity have lower rates of morbidity and mortality than those who do not. Serious games oriented to physical activity can offer an attractive and sustainable alternative or supplement traditional exercise modes [5].

Research has verified the relationship between physical activity and serious video games by providing entertainment and satisfactory experiences for the benefit of the health of the practitioner. Physical activity in combination with serious video games is a feasible and effective strategy to improve the upper and lower body, flexibility, balance and agility in older adults. As in [42], our main contribution is the insertion of new technologies but in Mexican adult environments, with cultural and customized activities, may boost the engagement with exercise; however we contribute further by also provide to additional health information to therapists or medical doctors and engaging the older adult with moderate activity, activity that impacts directly in their well-being. In future work, the proposed design will be evaluated with a set of participants between 60 and 80 years old to understand adherence to technology and health improvements. Also, this implementation will allow us to verify the impact on the older adults' health and perceived well-being and the inclusion of galvanic skin sensors to incorporate identification of emotional states while playing.

Acknowledgements PRODEP México under the award number UACH-PTC-370.

\section{References}

[1] Agmon, M., Perry, C. K., Phelan, E., Demiris, G., and Nguyen, H. Q. A pilot study of wii fit exergames to improve balance in older adults. Journal of geriatric physical therapy 34, 4 (2011), 161-167.

[2] Bateni, H. Changes in balance in older adults based on use of physical therapy vs the Wii Fit gaming system: a preliminary study. Physiotherapy 98, 3 (sep 2012), 211-216.

[3] Bauman, A., Merom, D., Bull, F. C., Buchner, D. M., and Fiatarone Singh, M. A. Updating the evidence for physical activity: Summative reviews of the epidemiological evidence, prevalence, and interventions to promote active aging. The Gerontologist 56, 2 (03 2016), S268-S280.

[4] Benetos, A., Adamopoulos, C., Bureau, J.-M., Temmar, M., Labat, C., Bean, K., Thomas, F., Pannier, B., Asmar, R., Zureik, M., Safar, M., and Guize, L. Determinants of accelerated progression of arterial stiffness in normotensive subjects and in treated hypertensive subjects over a 6-year period. Circulation 105, 10 (mar 2002), 1202-1207.

[5] Bock, B., Dunsiger, S., Ciccolo, J., Serber, E., Wu, W., Tilkemeier, P., Walaska, K., and Marcus, B. Exercise videogames, physical activity, and health: Wii heart fitness: A randomized clinical trial. American Journal of Preventive Medicine 56 (4) (2019), 501-511.

[6] Brox, E., Evertsen, G., 'Asheim Olsen, H., HorsFraile, S., and Browne, J. Experience with a $3 \mathrm{~d}$ kinect exergame for elderly, 2015.

[7] Chacon-Serna, M. J., Quino-' Avila, A. C., and' VallejoCastillo, L. F. Capacidad funcional del anciano relacionada con la actividad f'isica. Revista Investigacio'n en Salud Universidad de Boyac'a 4, 1 (jul 2017), 86.

[8] Chao, Y.-Y., Scherer, Y. K., and Montgomery, C. A. Effects of using nintendo wii ${ }^{\mathrm{TM}}$ exergames in older adults: a review of the literature. Journal of aging and health 27, 3 (2015), 379402.

[9] Charmaz, K. Constructionism and the Grounded Theory Method. In Handbook of Constructionist Research, J. A. Holstein and J. F. Gubrium, Eds. Guilford Publications, 2013, ch. 20, p. 832.

[10] Cornejo, R., Hernandez, D., Tentori, M., and Favela, J. Casual gaming to encourage active ageing. IEEE LATIN AMERICA TRANSACTIONS (Revista IEEE America Latina) 13 (6) (2015), 1940-1950. 
[11] Estadella, C., Vazquez, S., and Oliveras, A. Rigidez arterial y riesgo cardiovascular. Hipertension y Riesgo Vascular 27, 5 (sep 2010), 203-210.

[12] Fund, I. M. Is latin america prepared for an aging population? Tech. rep., International Monetary Fund, 2018.

[13] Garcia, J. A., Navarro, K. F., Schoene, D., Smith, S. T., and Pisan, Y. Exergames for the elderly: Towards an embedded kinect-based clinical test of falls risk. In HIC (2012), pp. 5157.

[14] Garcia, J. A., Pisan, Y., Tan, C. T., and Navarro, K. F. Assessing the Kinect's Capabilities to Perform a Time-Based Clinical Test for Fall Risk Assessment in Older People. In Entertainment Computing - ICEC 2014 (Berlin, Heidelberg, 2014), Y. Pisan, N. M. Sgouros, and T. Marsh, Eds., Springer Berlin Heidelberg, pp. 100-107.

[15] Gerling, K., Livingston, I., Nacke, L., and Mandryk, R. Fullbody motion-based game interaction for older adults. In Proceedings of the SIGCHI Conference on Human Factors in Computing Systems (New York, NY, USA, 2012), CHI '12, ACM, pp. 1873-1882.

[16] Gerling, K. M., Schild, J., and Masuch, M. Exergame design for elderly users: The case study of silverbalance. In Proceedings of the 7th International Conference on Advances in Computer Entertainment Technology (New York, NY, USA, 2010), ACE'10, ACM, pp. 66-69.

[17] Gopura, R., Kiguchi, K., and Horikawa, E. A study on human upper-limb muscles activities during daily upper-limb motions. International Journal of Bioelectromagnetism 12, 2 (2010), 54-61.

[18] Harley, D., Fitzpatrick, G., Axelrod, L., White, G., and McAllister, G. Making the wii at home: game play by older people in sheltered housing. In Symposium of the Austrian HCI and Usability Engineering Group (2010), Springer, pp. 156176.

[19] Leoncini, G., Ratto, E., Viazzi, F., Vaccaro, V., Parodi, A., Falqui, V., Conti, N., Tomolillo, C., Deferrari, G., and Pontremoli, R. Increased ambulatory arterial stiffness index is associated with target organ damage in primary hypertension. Hypertension (Dallas, Tex. : 1979) 48, 3 (sep 2006), 397-403.

[20] Lozano, R., Gomez-Dantes, H., Garrido-Latorre, F., JimenezCorona, A., Campuzano-Rincon, J. C.,' Franco-Marina, F., Medina-Mora, M. E., Borges, G., Naghavi, M., Wang, H., et al. Burden of disease, injuries, risk factors and challenges for the health system in mexico. Salud publica de Mexico 55, 6 (2013), 580-594.

[21] Mendizabal, M. Envejecimiento activo: un cambio de paradigma sobre el envejecimiento y la vejez. Aula Abierta 47, 1 (2018), 45-54.

[22] Milan, A., Tosello, F., Fabbri, A., Vairo, A., Leone, D., Chiarlo, M., Covella, M., and Veglio, F. Arterial stiffness: from physiology to clinical implications. High blood pressure \& cardiovascular prevention : the official journal of the Italian Society of Hypertension 18, 1 (mar 2011), 1-12.

[23] Millasseau, S. C., Kelly, R. P., Ritter, J. M., and Chowienczyk, P. J. Determination of age-related increases in large artery stiffness by digital pulse contour analysis. Clinical science (London, England : 1979) 103, 4 (oct 2002), 371-377.
[24] Neufeldt, C. Wii play with elderly people. Enhancing Interaction Spaces by Social Media for the Elderly 6, 3 (2009), $50-59$.

[25] Of Health, U. D., and Services, H. Physical Activity Guidelines for Americans, 2nd ed. Office of Disease Prevention and Health Promotion, 2018.

[26] Organization, W. H. Active aeging: a policy framework. Tech. rep., World Health Organization, Madrid, Spain, 2002.

[27] Organization, W. H. World report on ageing and health. Tech. rep., World Health Organization, 2015.

[28] Organization, W. H. Ageing and health. Tech. rep., World Health Organization, 2018.

[29] O’Loughlin, E., Dugas, E., Sabiston, K., and O'Loughlin, J. Prevalence and correlates of exergaming in youth. Pediatrics 130 (5) (2012), 806-814.

[30] Padial, M., Pinzon, S., Navarro, B., San Juan, P.,' Ruiz, J., and Espinosa, J. M. Implantacion efectiva de la Cuadruple Helice basada en el Modelo de Innovacion en envejecimiento activo. Gaceta Sanitaria 33, 5 (sep 2019), 491-494.

[31] Riano, M., Moreno, J., Echevarría, L., Rangel, L., and Sanchez, J. Condición física funcional y riesgo de caídas en adultos mayores. Revista Cubana de Investigaciones Biomédicas 37, 3 (2018), 1-10.

[32] Rodas, G., Pedret, C., Ramos, J., and Ort'1s, L. Heart rate variability: Definition, measurement and clinical relation aspects (part II). Archivos de Medicina del Deporte 25 (2008), 119-127.

[33] Rojas, A. M., Cebada, R. T., Cano, M. S., and Chacon, A. B.' Arteriosclerosis. patogenia de la arteriosclerosis. mecanismos celulares y moleculares implicados en la aterog'enesis. Medicine-Programa de Formaci'on M'edica Continuada Acreditado 9, 38 (2005), 2495-2505.

[34] Rosen, J., Perry, J. C., Manning, N., Burns, S., and Hannaford, B. The human arm kinematics and dynamics during daily activities-toward a 7 dof upper limb powered exoskeleton. In ICAR'05. Proceedings., 12th International Conference on Advanced Robotics, 2005. (2005), IEEE, pp. 532-539.

[35] Samad, R., Bakar, M. Z. A., Pebrianti, D., Mustafa, M., and Abdullah, N. R. H. Elbow flexion and extension rehabilitation exercise system using marker-less kinect-based method. International Journal of Electrical \& Computer Engineering (2088-8708) 7, 3 (2017).

[36] Saquib, N., Papon, M. T. I., Ahmad, I., and Rahman, A. Measurement of heart rate using photoplethysmography. In 2015 International Conference on Networking Systems and Security (NSysS) (Jan 2015), pp. 1-6.

[37] Sato, K., Kuroki, K., Saiki, S., and Nagatomi, R. Improving walking, muscle strength, and balance in the elderly with an exergame using kinect: A randomized controlled trial. Games for health journal 4, 3 (2015), 161- 167.

[38] Tarvainen, M. P., Lipponen, J., Niskanen, J.-P., and RantaAho, P. O. USER' S GUIDE HRV (Ver. 3.0.2) Standard, 2017.

[39] Tsipouras, M. G., and Fotiadis, D. I. Automatic arrhythmia detection based on time and time-frequency analysis of heart rate variability. Computer methods and programs in biomedicine 74, 2 (may 2004), 95-108. 
[40] Velarde, M., Perugachi, E., Romero, D., Sappa, A., and Vintimilla, B. Análisis del movimiento de las extremidades superiores aplicado a la rehabilitación física de una persona usando tecnicas de vision artificial. Revista Tecnologica ESPOL 28 (1) (2015), 1-7.

[41] Velazquez, A., Martınez-Garcia, A. I., Favela, J., and Ochoa, S. F. Adaptive exergames to support active aging: An action research study. Pervasive and Mobile Computing 34 (2017), $60-78$.
[42] Yeh, T., Pai, F., and Jeng, M. The factors affecting older adults' intention toward ongoing participation in virtual reality leisure activities. International Journal of Environmental Research and Public Health 16 (2019), 333-345.

[43] Young, W., Ferguson, S., Brault, S., and Craig, C. Assessing and training standing balance in older adults: a novel approach using the 'nintendo wii'balance board. Gait \& posture 33, 2 (2011), 303-305.

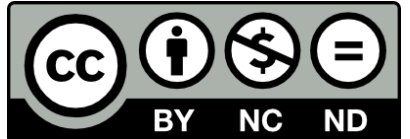

(C) 2020 by the authors. This work is licensed under the Creative Commons AttributionNonCommercial-NoDerivatives 4.0 International License. To view a copy of this license, visit http://creativecommons.org/licenses/by-nc-nd/4.0/ or send a letter to Creative Commons, PO Box 1866, Mountain View, CA 94042, USA. 